\title{
AN OPEN BOUNDARY CONDITION FOR APPLICATION IN NUMERICAL COASTAL MODELS
}

\author{
Peifeng $\mathrm{Ma}^{1}$ and Ole Secher Madsen ${ }^{2}$
}

\begin{abstract}
Open boundaries (OBs) are usually unavoidable in numerical coastal circulation simulations. At OBs, appropriate open boundary conditions (OBCs) are required and a good $\mathrm{OBC}$ should be able to let outgoing waves freely pass to the exterior of a computational domain without creating reflections at the OBs. In the present study, a methodology has been developed to predict two parameters, phase speed $c_{r}$ and decay time $T_{f}$, in a standard OBC formulation, so that the $\mathrm{OBC}$ is significantly improved compared to commonly used existing OBCs with specified $\mathrm{c}_{\mathrm{r}}$ and $\mathrm{T}_{\mathrm{f}}$. For the conditions where wave period is unknown, the $\mathrm{OBC}$ with approximated $\mathrm{c}_{\mathrm{r}}$ and $\mathrm{T}_{\mathrm{f}}$ may be applied and a test reveals that this $\mathrm{OBC}$ is able to yield good results in typical coastal flow conditions. In addition, a Swing-Door Boundary Condition (SDBC) is proposed and tested for application at an offshore open boundary where both incoming and outgoing waves exist.
\end{abstract}

Keywords: open boundary condition, bottom friction, phase speed, decay time, swing door boundary condition, numerical coastal model

\section{INTRODUCTION}

In most numerical simulations in coastal waters, open boundaries are always present and therefore an appropriate open boundary condition (OBC) must be applied. This OBC should be able to prevent any reflection of outgoing waves generated at the open boundary. Due to small water depth, the bottom friction may play a very important role in wave propagation in coastal waters. Thus, the bottom friction influences should be taken into account in the open boundary condition. Chapman (1985) summarized some commonly used open boundary conditions and tested their capability to prevent reflections, such as clamped boundary condition, Gravity-wave radiation boundary condition, the partially clamped boundary condition (Blumberg and Kantha, 1985), Orlanski radiation boundary condition (Orlanski, 1976), and sponge plus Orlanski radiation boundary condition, etc.. Among these open boundary conditions, only the partially clamped boundary condition introduced by Blumberg and Kantha (1985) has considered bottom friction effect by introducing a decay time of $T_{f}$ which, based on a test case, was suggested by the authors to be taken as 4hours. This open boundary condition is also used in the coastal ocean model FVCOM (Chen et al., 2006) with a specified decay time of $T_{f}=3$ hours.

It is known that, besides inducing wave attenuation, the bottom friction in coastal waters also reduces the phase speed $c_{r}$. However, this effect is not considered in the study of Blumberg and Kantha (1985) and in FVCOM, where $c_{\mathrm{r}}$ is taken as $\sqrt{g h}$. Clearly, both specified $c_{\mathrm{r}}$ and $\mathrm{T}_{\mathrm{f}}$ should be related to flow and bottom friction conditions rather than fixed values, as suggested by the study of Blumberg and Kantha (1985) and in FVCOM. Additionally, when simulating wave propagation from the offshore region towards the coast, one may have both incoming waves and outgoing reflected waves from the coast at the same offshore open boundary. Thus special treatment needs to be made at this type of open boundaries to avoid reflected waves being reflected back into the computational domain.

The objective of the present study is to develop a methodology for the prediction of $c_{r}$ and $T_{f}$ and propose an $\mathrm{OBC}$ at an offshore open boundary where both incoming and outgoing waves are present.

\section{FORMULATION}

Here we consider one dimensional shallow water equation described in terms of surface elevation $\eta$ and volume transport rate $q$

$$
\begin{aligned}
& \frac{\partial \eta}{\partial t}+\frac{\partial q}{\partial x}=0 \\
& \frac{\partial q}{\partial t}+\frac{\partial U q}{\partial x}=-g(h+\eta) \frac{\partial \eta}{\partial x}-\frac{\tau_{b}}{\rho}
\end{aligned}
$$

\footnotetext{
${ }^{1}$ Postdoctoral Associate, Singapore-MIT Alliance for Research and Technology, S16-05-08, 3 Science Drive, Singapore, 117543, mpf@smart.mit.edu

${ }^{2}$ Donald and Martha Harleman Professor, Parsons Laboratory, Massachusetts Institute of Technology, Room 48216C, 15 Vassar Street, Cambridge, MA, 02139, USA, osm@mit.edu
} 
where $q=U(h+\eta)$ is the volume transport rate with $h$, the still water depth, $U$ the depth-averaged velocity, and $\tau_{b}$ is the bed shear stresses which can be related to velocity $U$ by a quadratic form

$$
\tau_{b} / \rho=C_{b}|U| U
$$

where $C_{b}$ is a dimensionless bottom friction factor. This friction factor is dependent on bottom roughness, $k_{n a}$, experienced by the current, $U$, as $C_{b}=\left[\kappa / \ln \left(11 h / k_{n a}\right)\right]^{2}$. The presence of wind waves can significantly enhance the bottom roughness, e.g. Grant and Madsen, 1979. Thus the value of $C_{b}$ can vary over a wide range in coastal waters. For example, in a coastal region with water depth of $h=20 \mathrm{~m}$ and typical conditions of waves, $T=8 \mathrm{~s}$ and $H=0.5 \sim 3 \mathrm{~m}$, current, $U=0.5 \mathrm{~m} / \mathrm{s}$, and sediment, grain size $d$ of $0.2,0.4$ and $0.6 \mathrm{~mm}$, bottom roughness can be estimated by a wave-currentsediment interaction model proposed by Madsen(2002) and therefore the friction factor $C_{b}$ can be calculated from the above formulation. The results in Figure 1 reveal that the friction factor is small in weak wave conditions where the bed is flat and significantly increased when ripples are present due to stronger wind waves. When the wave height increases further, very strong near-bed flow may wash out ripples and therefore bottom friction factor is smaller than for rippled bed conditions, e.g. the case with grain size of $0.2 \mathrm{~mm}$ in Figure 1. In these typical coastal conditions, the estimated bottom friction factor, as shown in Figure 1, varies from $C_{b}=0.93 \times 10^{-3}$ corresponding to weak flow and fine sediment condition to $C_{b}=6.92 \times 10^{-3}$ for strong wave and coarse sediment condition.

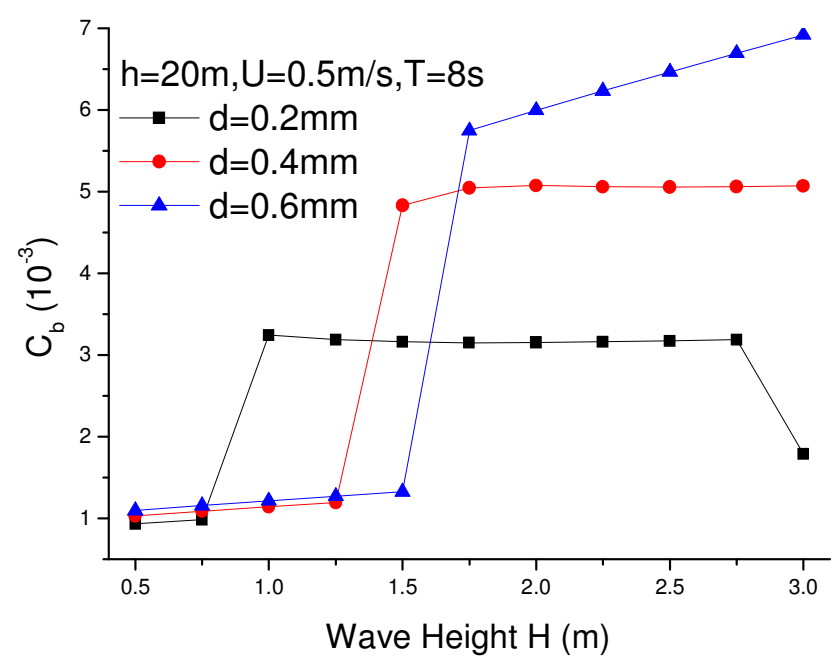

Figure 1 Bottom friction factor in typical coastal conditions

As introduced by Blumberg and Kantha (1985), a standard formulation of OBC in coastal waters in terms of free surface elevation can be expressed by

$$
\frac{\partial \eta}{\partial t}+c_{r} \frac{\partial \eta}{\partial x}=-\frac{\eta}{T_{f}}
$$

Where $c_{r}=$ the real part of wave phase speed at the open boundary and $T_{f}=$ the time scale of wave damping which should be related to bottom friction.

The OBC (4) can be reduced to other commonly used OBCs by choosing different combinations of $c_{r}$ and $T_{f}$. The radiation gravity wave condition can be obtained for $c_{r}=\sqrt{g h} \& T_{f}=\infty$ with $g=$ $9.81 \mathrm{~m} / \mathrm{s}^{2}$, the gravity acceleration. The Orlanski (1976) boundary condition is obtained if $T_{f}=\infty$ and the phase speed $c_{r}$ is computed by numerical results at interior points and in previous time steps as $c_{r}=-(\partial \eta / \partial t) /(\partial \eta / \partial x)$. If $T_{f}=0$, the boundary condition (4) becomes a clamped condition, $\eta=0$. The OBC (4) has been employed by Blumberg and Kantha (1985) in simulating wind-driven flow in a continental shelf sea and also by a community coastal ocean model, FVCOM (Chen et al., 2006). In both studies, the phase speed is computed by $c_{r}=\sqrt{g h}$, whereas the decay time $T_{f}=4$ hours is suggested by Blumberg and Kantha's study based on numerical tests and $T_{f}=3$ hours is the default value in FVCOM. 
Having the standard OBC (4), we can test the performance of some of the commonly used OBCs: the one used in Blumberg and Kantha (BK OBC) and the gravity wave open boundary condition (GW OBC). The test is carried out in an open channel with length of $L=80 \mathrm{~km}$ and still water depth of $h=20 \mathrm{~m}$. A semi-diurnal tidal wave with period of $T=12.4$ hours, the most common long wave in coastal waters, is prescribed at the upstream open boundary $x=0$ as $\eta=a_{0} \sin \omega t$ with $a_{0}=1 \mathrm{~m}$ and GW OBC or BK OBC is applied at the downstream open boundary at $x=L=80 \mathrm{~km}$. The Princeton Ocean Model (POM) is employed to carry out all tests in the present study. In this test, a grid size of $\Delta x=2 \mathrm{~km}$ and time step of $\Delta \mathrm{t}=12 \mathrm{~s}$ are used. Two types of bottom friction conditions are considered in the test: one is low friction condition with $C_{b}=0.001$ and the other is high friction condition with $C_{b}=0.008$. A numerical simulation in a very long channel with length $L=6000 \mathrm{~km}$ for each friction condition is conducted to provide a no-reflection solution. The amplitudes of elevation along the channel, which are computed by $a=\left(\eta_{\max }-\eta_{\min }\right) / 2$ with the maximum elevation $\eta_{\max }$ and minimum elevation $\eta_{\min }$ obtained after periodic steady state is reached, are plotted in Figure 2.

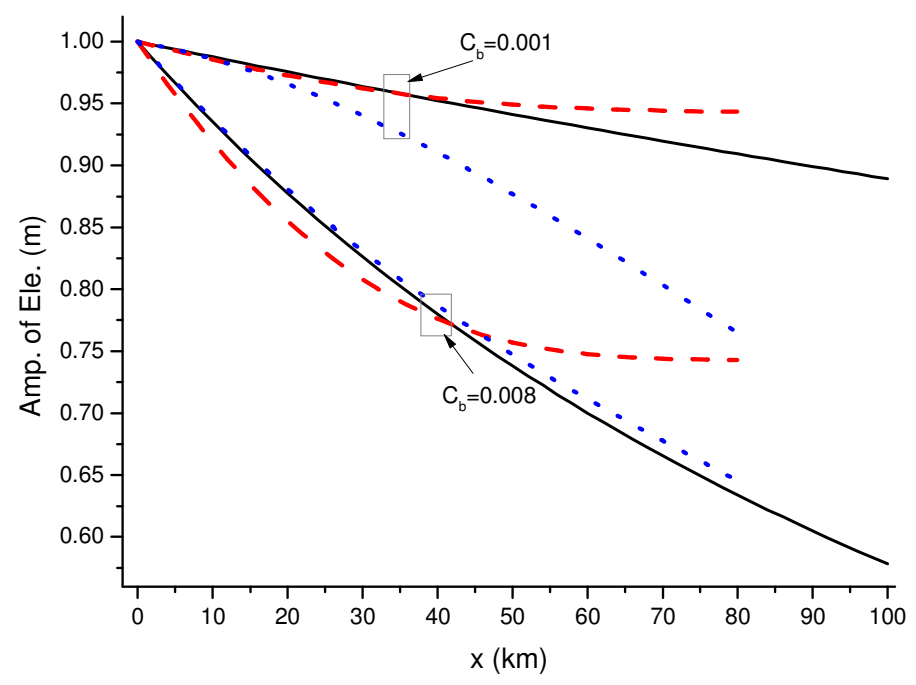

Figure 2 Amplitudes of surface elevation along the channel corresponding to long channel solution (solid), GW OBC (dashed) and BK OBC (dotted) with incident wave amplitude of $a_{0}=1 \mathrm{~m}$.

It can be seen from Figure 2 that the results with GW OBC show fairly good agreement with the long channel solution for the low and very poor agreement for the high friction condition, with $3.7 \%$ and $17.2 \%$ reflections at $x=L$ for the two conditions, respectively. On the other hand, the BK OBC works quite well for high and very poorly for the low friction condition, with $1.8 \%$ and $-15.9 \%$ reflections at $x=L$ for the two friction conditions, respectively. These results suggest that GW OBC and $\mathrm{BK}$ OBC can only be used for a certain condition and this may be caused by poorly specified values of $c_{r}$ and $T_{f}$ in GW OBC and BK OBC. This implies that good agreement should be possible for both friction conditions if we can find a way to predict the parameters $c_{r}$ and $T_{f}$ in OBC (4).

To predict the two parameters, we consider a small amplitude wave problem in a domain with constant depth of $h$ so that the nonlinear terms in Equations (2) and (3) can be neglected. We also assume a linear relation between the bottom shear stress and the volume transport rate as

$$
\tau_{b} / \rho=R q
$$

For $R=R(t)$, the linearized equation can be written

$$
\frac{\partial^{2} \eta}{\partial t^{2}}-g h \frac{\partial^{2} \eta}{\partial x^{2}}+R \frac{\partial \eta}{\partial t}=0
$$

From (3) and (5), the linear friction factor $R$ can be related the nonlinear fiction factor $C_{b}$ through

$$
R=C_{b}|U| / h
$$


Obviously, (7) gives a friction factor $R$ which is varying both in time and space. For convenience of derivation, we may replace $|U|$ in (7) by a constant, such as the amplitude of $|U|$ or the periodaveraged value of $|U|$, so that the friction factor $R$ becomes a constant.

We assume equation (6) has a progressive wave solution

$$
\eta=A e^{i(k x-\omega t)}
$$

Introduction of (8) into (6) gives

$$
k=k_{r}+i k_{i}= \pm \frac{\omega}{\sqrt{g h}} \sqrt{1+i R / \omega}
$$

Where "+" represents a wave propagating in $+x$ direction and "-" represents a wave propagating in $-x$ direction. In the present study, we just consider waves propagating in $+x$ direction. Thus, the real and imaginary parts of the wave number read

$$
\begin{aligned}
& k_{r}=\frac{\omega}{\sqrt{g h}} \sqrt{\frac{\sqrt{1+R^{2} / \omega^{2}}+1}{2}} \\
& k_{i}=\frac{\omega}{\sqrt{g h}} \sqrt{\frac{\sqrt{1+R^{2} / \omega^{2}}-1}{2}}
\end{aligned}
$$

Thus, the progressive wave (8) can be re-written as

$$
\eta=a_{0} \cdot e^{-k_{i} x} \cdot e^{i\left(k_{r} x-\omega t\right)}=a e^{i\left(k_{r} x-\omega t\right)}
$$

Where $a=a_{0} \cdot e^{-k_{i} x}$ is the attenuating wave amplitude due to bottom friction, with $a_{0}$ the amplitude at $x=0$.

Now, we have the analytical solution for attenuating long waves and may substitute this solution into the OBC formulation given by (4) to obtain

$$
\begin{aligned}
& c_{r}=\frac{\omega}{k_{r}}=\sqrt{g h} / \sqrt{\frac{\sqrt{1+R^{2} / \omega^{2}}+1}{2}} \\
& T_{f}=\frac{k_{r}}{\omega k_{i}}=\sqrt{\frac{\sqrt{1+R^{2} / \omega^{2}}+1}{\sqrt{1+R^{2} / \omega^{2}}-1}} / \omega
\end{aligned}
$$

Apparently the values of $c_{r}$ and $T_{f}$ depend on the wave period and the bottom friction conditions. In some extreme conditions, the formulations of $c_{r}$ and $T_{f}$ in (12a) and (12b) can be significantly simplified. Thus, for $R / \omega \ll 1,(12 \mathrm{a})$ and $(12 \mathrm{~b})$ can be approximated by

$$
\begin{aligned}
& c_{r} \approx \sqrt{g h} \\
& T_{f} \approx 2 / R
\end{aligned}
$$

In this relatively low friction or short period wave case, the variable $\omega$ drops out from the formulations. This means that the OBC (4) can be applied without the wave period being known a priori. This may be very useful since the typical wave period is often unknown for realistic coastal flow conditions.

On the other hand, if $R / \omega \gg 1$, e.g. long period motion with very strong frictional effects, (12a) and $(12 b)$ reduce to

$$
\begin{aligned}
& c_{r} \approx \sqrt{g h} \cdot \sqrt{2 /(R / \omega)} \\
& T_{f} \approx 1 / \omega
\end{aligned}
$$

Essentially, the simpler formulae (14a) and (14b) do not simplify the problem as all variables remain in the formulations.

Based on (12a) and (12b), the values of non-dimensional speed and decay time, $c_{r} / \sqrt{g h}$ and $T_{f} / T$ with $T=2 \pi / \omega$, are computed for $R / \omega$ ranging from 0.01 to 100 and plotted in Figures $3 \mathrm{a}$ and $3 \mathrm{~b}$. The parameters computed from the simplified formulae (13a), (13b), (14a) and (14b) are also shown in the figures. Apparently, the simplified formulae (13a-b) and (14a-b) provide good approximations for very small and very large value of $R / \omega$, respectively. And $c_{r} / \sqrt{g h}$ and $T_{f} / T$ computed by (13a) and (13b) 
are reasonably good for $R / \omega$ up to $O(1)$ compared to those obtained from (12a) and (12b) as shown in Figures $3 \mathrm{a}$ and $3 \mathrm{~b}$.

In coastal waters, the typical order of magnitudes of relevant parameters in the above formulations are $h \sim O(10 \mathrm{~m}), U \sim O(1 \mathrm{~m} / \mathrm{s}), C_{b} \sim O\left(10^{-3}\right)$ and $\omega \sim O\left(10^{-4} \mathrm{~s}^{-1}\right)$. Thus the order of magnitude of $R / \omega$ is $O(1)$. This implies that one needs to be careful when using the approximate formulations of $c_{r}$ and $T_{f}$ in numerical coastal simulations. For example, for water depth of $20 \mathrm{~m}$, semi-diurnal tide with velocity amplitude of $|U| \sim 1 \mathrm{~m} / \mathrm{s}$ and friction coefficient of $C_{b}=0.005$, the value of $R / \omega$ with $R$ computed from ( 7 ) is about 1.78 and therefore $c_{r} / \sqrt{g h} \approx 0.8$ and $T_{f} \approx 0.27 T=3.3$ hours, and the approximation (13b) gives $T_{f}=2 / R \approx 2.2$ hours and (14b) gives $T_{f}=T / 2 \pi \approx 2.0$ hours.
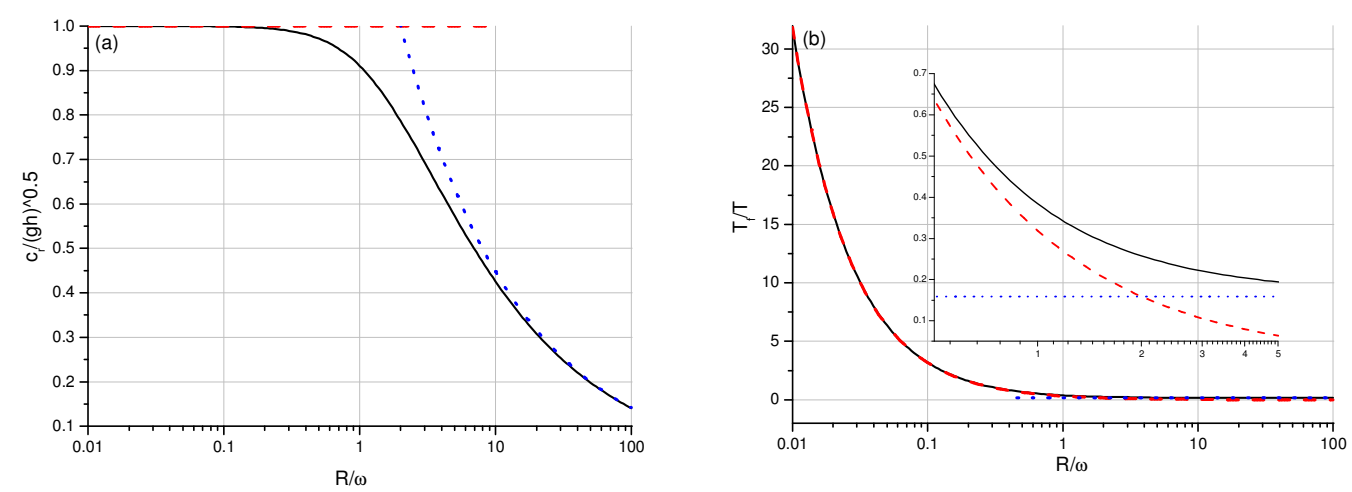

Figure 3 Variations of non-dimensional parameters with ratio $R / \omega$ for exact solution (solid), approximation for $R / \omega \ll 1$ (dashed) and approximation for $R / \omega \gg 1$ (dotted). (a) Phase speed $c_{r} / \sqrt{g h}$ (b) Decay time $T_{f} / T$

\section{APPLICATION TO TIDAL DOMINANT CONDITIONS}

Having predicted $c_{r}$ and $T_{f}$, we re-run the test case in Figure 2 with OBC (4). To be consistent with the derivation which assumed a constant value of $R$, the maximum velocity at $x=L=80 \mathrm{~km}$ obtained from a long channel solution is chosen to obtain the constant value of $R$ as $R=C_{b}\left|U_{\max }\right| / h$ and this $O B C$ is referred to as $\underline{O B C 1}$. Although consistent with the derivation of (12a) and (12b), this is clearly inconvenient, especially for simulations in real coastal waters where we may not be able to obtain a representative velocity from a simulation in a very large domain. Alternatively, we may calculate $c_{r}$ and $T_{f}$ in a very convenient way by using the instantaneous velocity at the closest interior grid points and taking this value for $|U|$ to compute $R$. We refer to this as $O B C 2$.

In the derivation of $c_{r}$ and $T_{f}$, linearity has been assumed. This actually requires the wave amplitude to be very small. However, waves may have finite amplitudes in most real conditions and nonlinear effects may not be negligible. In the case of large waves, we may modify OBC (4) by including some nonlinear effects related to elevation and velocity, including replacing the still water depth $h$ by the total water depth, $h+\eta$, and replacing phase speed $c_{r}$ by that for nonlinear waves superimposed on a current. Hence the factor $R$ and the phase speed $c_{r}$ are evaluated from

$$
\begin{aligned}
& R=C_{b}|U| /(h+\eta) \\
& c_{r}=\frac{\omega}{k_{r}} \sqrt{(h+\eta) / h}+U=\sqrt{g(h+\eta)} / \sqrt{\frac{\sqrt{1+R^{2} / \omega^{2}}+1}{2}}+U
\end{aligned}
$$

The decay time $T_{f}$ is computed by (12b) based on the modified friction factor given by (15a). The nonlinear characteristic analysis suggests that, with $c_{r}$ given by (15b), the calculation of $T_{f}$ by using (12b) is accurate enough for most conditions unless both $\eta / h$ and $R / \omega$ are very large. In (15b) the value of $U$ is obtained as in $O B C 2$, i.e. from the nearest interior grid point. For convenience, the OBC with these nonlinear effects included is referred to as $O B C 3$.

The predicted amplitudes of elevation along the channel from $\underline{O B C 1}$ and $\underline{O B C 2}$ for the incident wave amplitude $a_{0}=1 \mathrm{~m}$ are shown in Figure $4 \mathrm{a}$ for three friction factors $C_{b}=0.001,0.0025$ 
and 0.008. To test $O B C 3$, we specify the semi-diurnal tidal wave with amplitude of $a_{0}=3 \mathrm{~m}$ at $x=0$ and apply $\underline{O B C 2}$ or $\underline{O B C 3}$ at $x=L=80 \mathrm{~km}$ to carry out simulations in the same domain as in the previous test. The amplitudes of elevation along the channel are shown in Figure $4 \mathrm{~b}$.
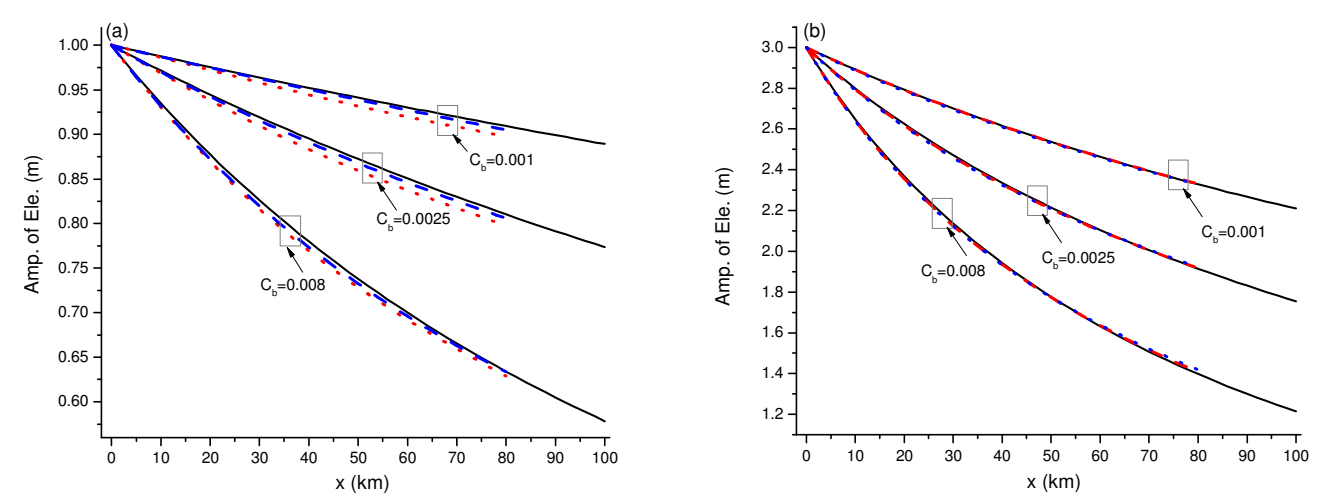

Figure 4 Wave amplitudes along the channel (a) Incident wave amplitude of $a_{0}=1 \mathrm{~m}$ with long channel solution (solid), $O B C 1$ (dotted) and $O B C 2$ (dashed); (b) Incident wave amplitude of $a_{0}=3 \mathrm{~m}$ with long channel solution (solid), $\underline{O B C 2}$ (dotted) and $\underline{O B C 3}$ (dashed).

As expected, the $\underline{O B C 1}$ with predicted $c_{r}$ and $T_{f}$ works very well for all of the three friction conditions shown in Figure $4 \mathrm{a}$. And the results show much better agreement with the long channel solutions than GW OBC and BK OBC in Figure 2. $O B C 1$ causes $-1.3 \%$ and $-0.8 \%$ reflections at $x=L$ for friction conditions of $C_{b}=0.001$ and 0.008 , respectively, as opposed to $3.7 \%$ and $17.2 \%$ for $\mathrm{GW}$ $\mathrm{OBC}$ and $-15.9 \%$ and $1.8 \%$ for BK OBC. The results corresponding to $O B C 2$ show even better agreements than those corresponding to $\underline{O B C 1}$, with $-0.5 \%$ and $-0.06 \%$ reflections at $x=L$ for friction factors of $C_{b}=0.001$ and 0.008 , respectively. This seems to be inconsistent with the derivation of $c_{r}$ and $T_{f}$ where $R$ was assumed constant. Nevertheless this should be theoretically reasonable if we look at the OBC (4) along the characteristic lines expressed by $d x / d t=c_{r}$ as $d \eta / d t=-\eta / T_{f}$. Numerically it can be expressed as $\Delta \eta / \Delta t=-\eta / T_{f}$ in which the elevation is the one at time $t$ and therefore the corresponding $T_{f}$ should be related to the velocity at the same time, i.e. $U(t)$. The better results associated with varying $R$ is very encouraging as we can obtain better results in a far more convenient way.

The calculations with $\underline{O B C 1}$ show that the predicted values of $c_{r}$ in this test varies only slightly for the three friction conditions, $c_{r} / \sqrt{g h}=0.99,0.98$ and 0.91 whereas the predicted values for $T_{f}$ are far more sensitive, $17.9,8.8$ and 4.7 hours for $C_{b}=0.001,0.0025$ and 0.008 , respectively. This confirms our conjecture for the reason behind the poor performance of GW and BK OBC shown in Figure 2, i.e. it is caused primarily by the poorly specified values for $T_{f}$. Actually the value of $T_{f}=$ 4hours in BK OBC happens to be close to the one predicted for the large friction condition, 4.7hours. And $T_{f}=17.9$ hours, as predicted for the low friction case, actually gives quite weak damping effect which is not much different from $T_{f}=\infty$ as assumed in GW OBC.

Surprisingly, it can be seen from Figure $4 \mathrm{~b}$ that the open boundary conditions, both $\underline{O B C 2}$ and $\underline{O B C 3}$, work very well for this large amplitude wave condition, with reflections at $x=L$ less than $1 \%$ for all the cases in Figure $4 \mathrm{~b}$. Further analysis of the time series of elevation suggests that the mean water elevation and mean velocity corresponding to $O B C 2$ and $O B C 3$ are quite different, with the mean values from $O B C 3$ much closer to the long channel solutions. These mean values are obtained by taking a period-average of elevation and velocity over the wave cycle from 130 to 142.4 hour. Some mean values at selected locations, i.e. mean velocity at $x=0,40 \mathrm{~km}$ and $80 \mathrm{~km}$, referred to as $\bar{U}_{0}, \bar{U}_{40}$, $\bar{U}_{80}$, and mean elevations at $x=40 \mathrm{~km}$ and $80 \mathrm{~km}$, referred to as $\bar{\eta}_{40}, \bar{\eta}_{80}$, for the wave amplitude of $3 \mathrm{~m}$ and friction factor of $C_{b}=0.001$ and 0.008 are listed in Table 1. Apparently, for both friction conditions, the mean values from $\underline{O B C 3}$ are much closer to the long-channel solutions than those from $\underline{O B C 2}$, e.g. the mean surface elevation at $x=L=80 \mathrm{~km}$ from $\underline{O B C 3}$ for $C_{b}=0.001$ has only a $-0.7 \%$ difference from the long channel solution whereas that from $\underline{O B C 2}$ has a $-59 \%$ difference. It can be also observed in Table 1 that the period-averaged velocities from $\underline{O B C 2}$ at $x=40 \mathrm{~km}$ and $80 \mathrm{~km}$ have 
positive values whereas those from long channel solutions and $\underline{O B C 3}$ are negative. This could be crucial if one is interested in predicting long term averaged flows, such as residual tidal flows. Some numerical investigations with a smaller wave, $a_{0}=1 \mathrm{~m}$, suggest that the performance of $\underline{O B C 2}$ relative to $\underline{O B C 3}$ is even worse as far as the mean water level and period-averaged velocity are concerned despite the smaller amplitude. These results indicate that $\underline{O B C 3}$ is superior to $\underline{O B C 2}$, especially as far as mean water level and return flow are concerned.

\begin{tabular}{|c|c|c|c|c|c|c|}
\hline \multicolumn{7}{|c|}{ Table 1 Period-averaged elevation and velocity along the channel } \\
\hline & & $\begin{array}{c}\bar{\eta}_{40} \\
(\mathrm{~cm})\end{array}$ & $\begin{array}{c}\bar{\eta}_{80} \\
(\mathrm{~cm})\end{array}$ & $\begin{array}{c}\bar{U}_{0} \\
(\mathrm{~cm} / \mathrm{s})\end{array}$ & $\begin{array}{c}\bar{U}_{40} \\
(\mathrm{~cm} / \mathrm{s})\end{array}$ & $\begin{array}{c}\bar{U}_{80} \\
(\mathrm{~cm} / \mathrm{s})\end{array}$ \\
\hline \multirow{3}{*}{$C_{b}=0.001$} & $\underline{\text { LONG }}$ & 9.74 & 15.91 & -9.87 & -7.15 & -5.15 \\
$a_{0}=3 m$ & $\underline{\text { OBC2 }}$ & 4.70 & 6.60 & 0.65 & 3.27 & 5.27 \\
& $\underline{\text { OBC3 }}$ & 9.35 & 15.80 & -10.76 & -8.06 & -6.02 \\
\hline \multirow{3}{*}{$C_{b}=0.008$} & $\underline{\underline{L O N G}}$ & 17.10 & 20.78 & -6.34 & -2.32 & -0.60 \\
$a_{0}=3 m$ & $\underline{\text { OBC2 }}$ & 11.43 & 10.67 & -3.53 & 0.58 & 2.35 \\
& $\underline{\text { OBC3 }}$ & 15.15 & 17.13 & -5.42 & -1.28 & 0.48 \\
\hline
\end{tabular}

\section{A TEST ON THE OBC WITH APPROXIMATE $c_{r}$ and $T_{f}$}

In the above tests, we have assumed wave period is known a priori so that parameters $c_{r}$ and $T_{f}$ can be computed via (12a) and (12b). This is feasible in some coastal areas where tidal flow is dominant. However, in some circumstances, we may not know the wave period, such as wind generated oscillations and storm surges in coastal waters. To handle such problems, we may use some approximate form of OBC. As discussed in Section 2, the leading terms of $c_{r}$ and $T_{f}$ in (13a) and (13b) for small values of $R / \omega$ are independent of wave period. Thus, we may apply (13a) and (13b) as an approximation when we do not have accurate information about wave period. The typical period of wind generated flow motion and storm surges is several hours. With this period, the value of $R / \omega=$ $C_{b}|U| /[(h+\eta) \omega]$ would have a value smaller than 1 for typical coastal conditions. Surely, there are some slowly varying motions in coastal waters which lead to a large value of $R / \omega$ and therefore make (13a) and (13b) invalid. To investigate how the value of $R / \omega$ affects the performance of the approximation of (13a) and (13b), we perform two tests here: one is for semi-diurnal tidal flow with period of $T=12$.4hours and amplitude of $a_{0}=1 \mathrm{~m}$ at $x=0$ which gives $R / \omega<1$ and the other is for slowly varying flow which has a period of $T=62$ hours and amplitude of $a_{0}=3 \mathrm{~m}$ at $x=0$ and gives $R / \omega>1$ for most of the time. As in previous tests, the elevation time series is specified at $x=$ 0 and the approximate values for $c_{r}$ and $T_{f}$ in the OBC are expressed by (13a) and (13b) and the OBC (we refer to it as $\underline{O B C 4}$ ) is applied at $x=80 \mathrm{~km}$. The results with $\underline{O B C 3}$ are also shown for comparison.

The amplitudes of elevation along the channel are shown in Figure $5 \mathrm{a}$ and $5 \mathrm{~b}$ for the tidal wave case and the slowly varying motion case, respectively. The time series of values of $R / \omega$ over approximately one wave cycle are shown in Figure $6 \mathrm{a}$ and $6 \mathrm{~b}$ for the two cases, respectively. Again, three types of friction conditions are considered, i.e. $C_{b}=0.001,0.0025$ and 0.008 . It can be seen from Figure $5 \mathrm{a}$ that the predicted wave amplitudes from $\underline{O B C 4}$ agree with the long channel solutions very well, which are very close to the solutions with $\underline{O B C 3}$. These good results can be expected as relatively small values of $R / \omega$ are obtained for this case. Time series of $R / \omega$ in Figure 6a suggest that $R / \omega<1$ is assured for most conditions except for high friction conditions with $C_{b}=0.008$. In fact, for the case with $C_{b}=0.008$, the value of $R / \omega$ is not small enough to assure the validity of $O B C 4$. The good result for this case may be partially due to the vanishing of the first order term in $R / \omega$ in the expansion of $T_{f}$ in terms of the small parameter $R / \omega$ as $1 / T_{f}=R / 2+O\left(R^{2} / \omega^{2}\right)$. 

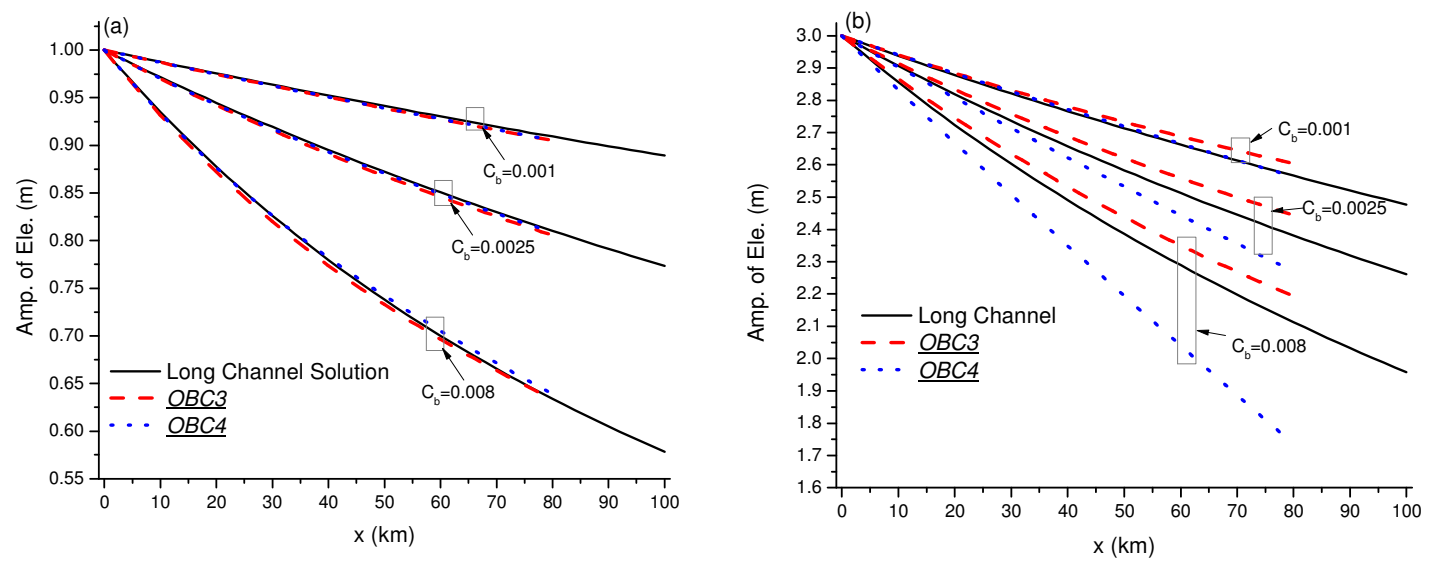

Figure 5 Elevation amplitudes along the channel for various OBCs. (a) Small amplitude M2 tide case with small $R / \omega$; (b) Large amplitude slowly varying motion case with large $R / \omega$.
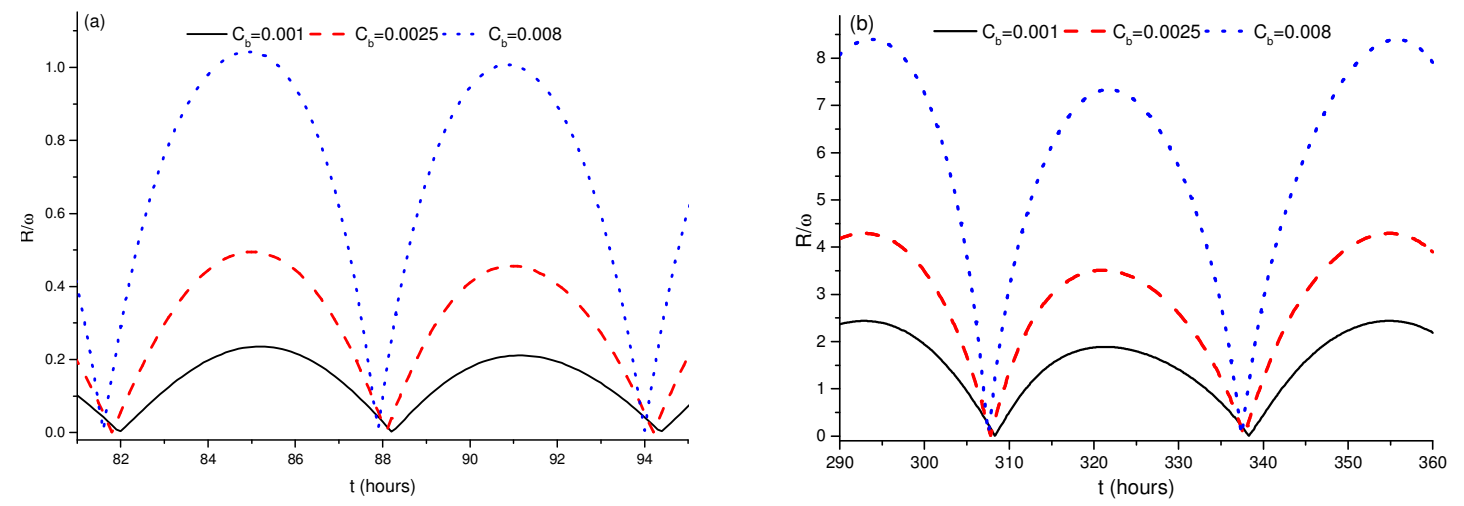

Figure 6 Time series of $R / \omega$ in about one wave cycle computed based on long channel solutions. (a) Small amplitude M2 tide case; (b) Large amplitude slowly varying motion case.

Based on the derivation, $\underline{O B C 4}$ should introduce errors if applied to conditions with $R / \omega>1$. In the test of slowly varying motion, the value of $R / \omega$ shown in Figure $6 \mathrm{~b}$ is greater than 1 even for the low friction condition with $C_{b}=0.001$ and as large as nearly 10 in the high friction condition with $C_{b}=0.008$. With these large values, one would expect very poor agreement compared with long channel solutions. However, the predictions with $\underline{O B C 4}$ in Figure 5b show quite good agreement with long channel solutions, especially for the case with $C_{b}=0.001$ and 0.0025 . Even in the high friction condition with $C_{b}=0.008$ where $R / \omega$ has values up to nearly 10 , the relative error at $x=80 \mathrm{~km}$ is only about $18 \%$. This is surprising and interesting. Further investigations suggest that the good results may be obtained because the errors caused by the inaccurate $c_{r}$ and $T_{f}$ somehow cancel out. In $\underline{O B C 4}$, the approximate value of $c_{r}$ from (13a) is larger than the more accurate value obtained from (12a) and $T_{f}$ from (13b) is smaller than the more accurate value obtained in $\underline{O B C 3}$ from (12b). Considering the $\mathrm{OBC}$ along the linear characteristic, $d x / d t=c_{r}$, the OBC $d \eta / d t=-\eta / T_{f}$ gives the solution along the characteristic as $\eta \propto \exp \left(-t / T_{f}\right)=\exp \left[-x /\left(c_{r} T_{f}\right)\right]$. From this, we can see that for a given travel distance $x$, the predicted wave amplitude will increase if phase speed $c_{r}$ is over-estimated and decrease if $T_{f}$ is under-predicted. This suggests that the good results in the case with large value of $R / \omega$ may actually be due to, at least partially, the canceling out of the errors induced by the inaccurate values of 
$c_{r}$ and $T_{f}$ in $\underline{O B C 4}$. Regardless of this coincidence, the $\underline{O B C 4}$ may be a reasonable choice in circumstances when we do not have any information on the dominant wave period of the motion.

\section{SWING-DOOR BOUNDARY CONDITION (SDBC)}

When simulating flows in continental shelf seas, another kind of problem may be encountered. Sometimes waves propagate from the offshore region towards the coast and the incident wave is known at an offshore location. In such a scenario, waves at this offshore location consist of both incoming waves and outgoing waves reflected by the coast. Therefore a good OBC at this offshore location should allow incoming waves to freely propagate into the domain and at the same time let the outgoing reflections pass out to the exterior without any reflection back into the computational domain. Here we propose an OBC to deal with this situation and refer to it as a Swing-Door Boundary Condition (SDBC). This SDBC is implemented in the following way.

Considering a one-dimensional problem, we assume an offshore open boundary at $x=0$ and the coast at $x=L$. The total elevation $\eta_{T}$ at any location inside the domain can be expressed as the sum of the incoming wave $\eta_{i}$ and the outgoing reflection $\eta_{r}$ created by the coast at $x=L$

$$
\eta_{T}=\eta_{i}+\eta_{r}
$$

The incoming wave $\eta_{i}$ at $x=0$ is known as

$$
\eta_{i}=a_{0} e^{i \omega t}
$$

To obtain the total surface elevation $\eta_{T}$ at $x=0$ for use in the numerical integration, one needs to know the reflected wave $\eta_{r}$ at $x=0$. Obviously, the OBC (4) can be applied to calculate the reflected wave $\eta_{r}$ at $x=0$, i.e.

$$
\frac{\partial \eta_{r}}{\partial t}+c_{r} \frac{\partial \eta_{r}}{\partial x}=-\frac{\eta_{r}}{T_{f}}
$$

where $c_{r}$ and $T_{f}$ can be computed by assuming linear friction condition at the first grid of the computational domain, $x=0 \sim \Delta x$. With this approximation, the linearized friction factor at $x=0 \sim \Delta x$ can be computed by

$$
R=C_{b}|U|_{x=\Delta x} /(h+\eta)
$$

where $\mathrm{U}$ is the total velocity at $x=\Delta x$ calculated by the numerical model. Since this reflected wave propagates in $-x$ direction, $c_{r}$ in (18) should in principle be negative. However, we choose it to be positive to be consistent with our sign convention for outgoing waves, and obtain $\partial \eta_{r} / \partial x$ numerically from $\partial \eta_{r} / \partial x=\left(\eta_{r, x=0}-\eta_{r, x=\Delta x}\right) / \Delta x$. With the linear friction condition in the first grid, the incoming wave $\eta_{i}$ at $x=\Delta x$ can be computed analytically by $\eta_{i}(x=\Delta x)=a_{0} \exp \left(-k_{i} \Delta x\right) \exp \left[i\left(k_{r} \Delta x-\omega t\right)\right]$ with $k_{r}$ and $k_{i}$ computed by (10a) and (10b) based on friction factor $R$ given by (19). Then the reflected wave $\eta_{r}$ at $x=\Delta x$ can be computed by subtracting the incoming wave from the total wave at $x=\Delta x$, i.e. $\eta_{r}(x=\Delta x)=\eta_{T}(x=\Delta x)-\eta_{i}(x=\Delta x)$. Having $\eta_{r}$ at $x=\Delta x$, the reflected wave $\eta_{r}$ at $x=0$ can be computed from (18). Eventually, the total elevation at $x=0$ is computed by (16).

For a linear problem, this SDBC should be exactly correct. However, if nonlinear effects are involved, it is approximate and hard to verify since we do not have an equivalent to the long channel solution used in the tests of the OBC. One way we can verify the SDBC is to check if there is evidence of reflection in the time series of elevation at a certain location, e.g. at the coast $x=L$. If the SDBC works perfectly, there will be no reflection generated at $x=0$ and the total wave at any location inside the domain is the combination of the incoming wave and the first reflected wave by the coast. Therefore the wave amplitude at the coast should be unchanged after the periodic steady state is reached. On the other hand, if just specifying the incoming wave at $x=0$, i.e. $\eta_{T}=\eta_{i}$, the offshore open boundary will act to fully reflect the outgoing wave and the wave will be reflected back and forth inside the domain. Consequently the wave amplitude at the coast will be varying. Particularly, if we select a domain length corresponding to resonance, the wave amplitude at the coast will keep on increasing. Therefore we can compare the time series of surface elevation at the coast from these two types of OBCs to check the performance of our SDBC.

In this test, we assume the incoming wave has a period of $T=2$ hours and the domain has a length of $L=0.75 \lambda$ with $\lambda$ the wavelength of the incoming wave so that resonance takes place for the OBC of $\eta_{T}=\eta_{i}$ at $x=0$. The following parameters are used in the tests: incoming wave amplitude of 
$a_{0}=1 \mathrm{~m}$; still water depth of $h=20 \mathrm{~m}$; channel length of $L=0.75 \lambda=76 \mathrm{~km}$; grid size of $\Delta x=1 \mathrm{~km}$; time step of $\Delta \mathrm{t}=2 \mathrm{~s}$; and friction factor $C_{b}=0.001,0.0025$ or 0.008 .

At this length of channel, it takes the incoming wave or reflected wave at $x=0$ about 1.5 hours to reach the coast at $x=L$. This means the wave amplitude at the coast, $x=L$, would be changed by reflections every 3 hours. Here we show the elevation time series at $x=L$ only for $C_{b}=0.001$ in Figure $7 \mathrm{a}$, since resonance effect is more pronounced for small frictional attenuation. It can be seen that the amplitude becomes nearly $2 \mathrm{~m}$ when the incident wave reaches the coast and is reflected back by the coast. The results in Figure 7a suggest that, if SDBC is applied, the wave amplitude changes very little. On the other hand, if applying $\eta_{T}=\eta_{i}$ at $x=0$, the wave amplitude increases after $\mathrm{t}=4$.5hours to be about $3 \mathrm{~m}$ and keeps increasing until the multi-reflected wave decays to near zero by the bottom friction at about $t=12$ hour. The nearly unchanged wave amplitude associated with the SDBC in Figure 7a suggests that the SDBC is able to let reflections created at $x=L$ leave the domain at $x=0$. The amplitude variation for different friction conditions are shown in Figure $7 \mathrm{~b}$ for the two boundary conditions. It can be observed clearly in Figure $7 \mathrm{~b}$ that the SDBC works very well for the purpose of preventing reflections at the offshore open boundary.

While testing the SDBC, it has been found that the mean water level may shift quite a lot if $\underline{O B C 2}$ is applied to the reflected wave $\eta_{r}$ at $x=0$ and the shift is very small if $O B C 3$ is used. This shift is not obvious if the resonance length is selected as in the above test. An example with channel length $L=0.85 \lambda$ and bottom friction factor of $C_{b}=0.0025$ shown in Figure 8, suggests that the mean water level is apparently shifted by about $0.5 \mathrm{~m}$ when linear form of $\mathrm{OBC}(\underline{O B C 2})$ is applied, which is relatively huge as the incident wave amplitude is only $1 \mathrm{~m}$, and the shift is very small if nonlinear effects are incorporated $(\underline{O B C 3})$. This is similar to the difference between $\underline{O B C 2}$ and $\underline{O B C 3}$ predictions of mean elevation and velocity discussed in the previous section.

The underlying reason for this is not clear to the authors, but the results indicate that $O B C 3$ appears to be the better choice.
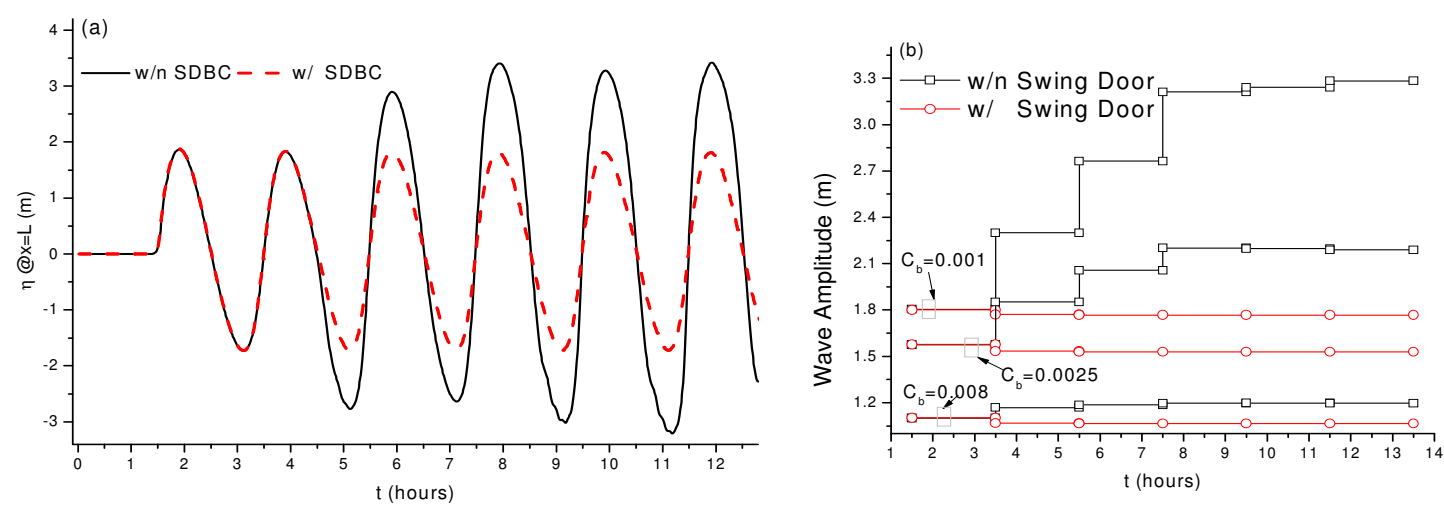

Figure 7 Tests on Swing-Door Boundary Condition in a channel with length of $L=0.75 \lambda$. (a) Time series of elevation at $\mathrm{X}=\mathrm{L}$ for small friction condition, $C_{b}=0.001$; (b) Variation of elevation amplitude at $\mathrm{X}=\mathrm{L}$ for various friction conditions. 


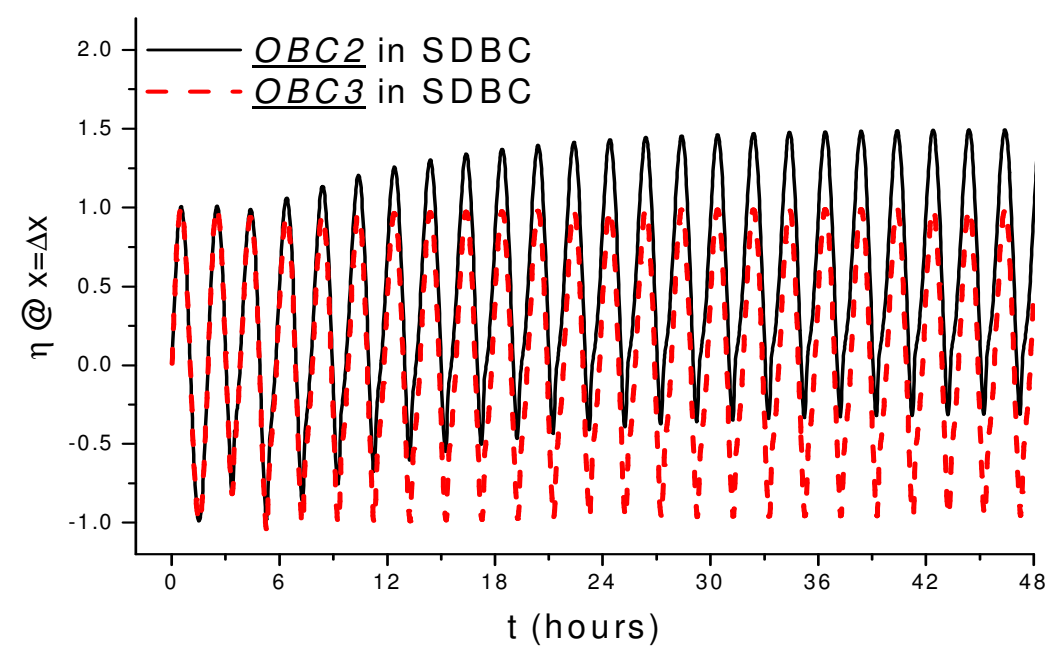

Figure 8 Elevation time series at $\mathrm{x}=\Delta \mathrm{x}$ in a channel with length of $L=0.85 \lambda$

\section{CONCLUSIONS}

In the present study, a methodology has been developed to predict the two key parameters in a standard open boundary condition, phase speed $c_{r}$ and decay time $T_{f}$, to significantly improve the performance of the boundary condition. Derived from linearized shallow water equations, the two parameters are dependent on the wave period and constant linearized friction factor. In accordance with the derivation, a test with linear friction factor computed by the maximum velocity is carried out and good results suggest that the open boundary condition $(\underline{O B C 1})$ is able to prevent most reflections at the open boundary. For convenience of numerical implementation, an open boundary condition $(O B C 2)$ with varying friction factor based on instantaneous velocity at the nearest interior grid point is proposed and the test shows $O B C 2$ is able to produce a even better results than the $O B C 1$. Furthermore, when including nonlinear terms in predicting $c_{r}$ and $T_{f}$, the open boundary condition $(\underline{O B C 3})$ is able to predict very good results for large wave conditions in which the nonlinear effects could be important. Meanwhile, the tests also suggest that the $\underline{O B C 3}$ can predict much better mean water levels and mean velocities over a wave period than $\underline{O B C 2}$. This is very important if one is concerned with return or residual flows.

Despite the good performance of the OBC, either one of $\underline{O B C 1}, \underline{O B C 2}$ or $\underline{O B C 3}$, it has a serious limitation, i.e. the wave period needs to be known a priori to predict $c_{r}$ and $T_{f}$ via (12a) and (12b). To remove this restriction, an $\mathrm{OBC}(\underline{O B C 4})$ with an approximate $c_{r}$ and $T_{f}$, (13a) and (13b), valid for $R / \omega \ll 1$ is tested for two types of coastal flows in which one is small amplitude tidal flow for which $R / \omega<1$ holds and the other is large-amplitude slowly varying motion, for which $R / \omega<1$ is violated. As expected, good performance is obtained for the small amplitude tidal flow case. And surprisingly, $\underline{O B C 4}$ only produces an error of amplitude less than about $18 \%$ for the case of large amplitude slowly varying motion even though the value of $R / \omega$ is as large as 10 . Investigations reveal that the effects of over-predicted $c_{r}$ and under-estimated $T_{f}$, resulting from the evaluation of these from the approximate expressions (13a and 13b), somehow cancel out. This result is very encouraging as $R / \omega$ is of $\mathrm{O}(1)$ in most typical coastal conditions. This indicates that we may, with some confidence, apply $\underline{O B C 4}$ in numerical coastal simulations when wave period is unknown.

To prevent reflections occurring at an offshore open boundary where both incoming wave and outgoing wave exist, a Swing-Door Boundary Condition (SDBC) is proposed. In this SDBC, the standard $\mathrm{OBC}$ is applied to the outgoing reflected wave which is separated from the total wave so that the incoming wave can freely propagate into the domain and at the same time outgoing reflections from the interior can pass out through the same open boundary without creating any reflection. A simple test suggests that the SDBC works very well for the purpose of preventing reflections at the offshore open boundary. 
The tests in the present study suggest that the $\underline{O B C 3}$ predicts very close wave amplitudes compared to $\underline{O B C 2}$ and both of $\underline{O B C 2}$ and $\underline{O B C 3}$ are convenient for numerical implementation. However, the $O B C 3$ predicts much better result as far as residual flow and mean water level are concerned as discussed in Section 3. Furthermore, a simple SDBC test shows that the mean water level near the offshore open boundary may shift remarkably when $O B C 2$ is applied and very little when $\underline{O B C 3}$ is used. This indicates that the $\underline{O B C 3}$ should be the best choice of OBC to apply in numerical coastal models if the dominant wave period is known.

Despite of the good performance of the $\mathrm{OBC}$ with predicted values for $c_{r}$ and $T_{f}$, there are some limitations of the present study. One of the limitations is that the tests are essentially one-dimensional problems, i.e. waves propagate in the normal direction to the open boundary. This is not the case in most real flows in coastal waters where waves may move across an open boundary at any angle. A methodology is being developed to incorporate the effects of oblique incidence at open boundaries so that the OBC can be applied to more realistic coastal conditions. Another restriction is that all of the tests in the present study are for simple periodic wave conditions which is an oversimplification for most flows encountered in coastal waters. Some tests are being undertaken for combined wave conditions where waves have different periods and amplitudes as well. Furthermore, Coriolis force may be very important in some large scale simulations. Hence investigation of its effect on the $\mathrm{OBC}$ is currently being pursued.

\section{ACKNOWLEDGMENTS}

The research described in this paper was funded in whole or in part by the Singapore National Research Foundation (NRF) through the Singapore-MIT Alliance for Research and Technology's (SMART) Center for Environmental Sensing and Modeling (CENSAM).

\section{REFERENCES}

Blumberg A. F. and Kantha L. H.. 1985. Open Boundary condition for circulation models, J. Hydraul. Eng., Vol. 111, No. 2, 237-255.

Chapman D. C. 1985. Numerical treatment of cross-shelf boundaries in a barotropic coastal ocean model, J. Phys. Oceanogr., Vol. 15, 1060-1075.

Chen, C., Beardsley, R. C., and Cowles, G. 2006. An unstructured grid, finite-volume coastal ocean model, FVCOM User Manual, Second Edition.

Grant, W. D. and Madsen, O. S. 1979. Combined wave and current interaction with a rough bottom, $J$. Geophys. Res., Vol. 84(C4), 1979-1808.

Madsen, O. S., 2002. Sediment Transport Outside the Surf Zone. Chapter III.6 in Coastal Engineering Manual, Part III Coastal Processes. (T. Walton, ed.), Engineer Manual 1110-2-1100, U.S. Army Corps of Engineers, Washington, DC.

Orlanski, I. 1976. A simple boundary condition for unbounded hyperbolic flows, J. Comput. Phys., Vol. 21, 251-269. 